\title{
CARACTERIZACIÓN METEOROLÓGICA DEL EPISODIO DE ALTA CONTAMINACIÓN EN MADRID DURANTE LA NAVIDAD DE 2016
}

Carlos Yagüe $^{(1)}$, Mariano Sastre ${ }^{(1)}$, Gregorio Maqueda ${ }^{(1)}$, Rafael Borge ${ }^{(2)}$, Begoña Artíñano $^{(3)}$, Francisco J. Gomez-Moreno ${ }^{(3)}$, Adolfo Narros ${ }^{(2)}$, Carlos RománCascón $^{(1)}$, Jon A. Arrillaga ${ }^{(1)}$, Marcos Barreiro ${ }^{(3)}$, Juan Manuel de Andrés ${ }^{(2)}$

(1) Departamento de Física de la Tierra y Astrofísica, Facultad de Ciencias Físicas, Universidad Complutense de Madrid, España (carlos@ucm.es)

(2) Dpto. de Ingeniería Química Industrial y Medioambiente, ETSII, Universidad Politécnica de Madrid, España ${ }^{(3)}$ Dpto. Medio Ambiente, CIEMAT, Madrid, España

Episodios de alta contaminación por dióxido de nitrógeno $\left(\mathrm{NO}_{2}\right)$ y otros contaminantes se pueden producir especialmente durante el otoño e invierno en la ciudad de Madrid (Artíñano et al., 2003), cuando tienen lugar condiciones meteorológicas adversas para la calidad del aire, como son la presencia de anticiclones persistentes (gran escala) que favorecen la subsidencia (Fig. 1), la generación de inversiones térmicas en las capas bajas de la atmósfera, capas de mezcla someras (Fig. 2) y en ocasiones vientos y turbulencia débiles en superficie (pequeña escala).

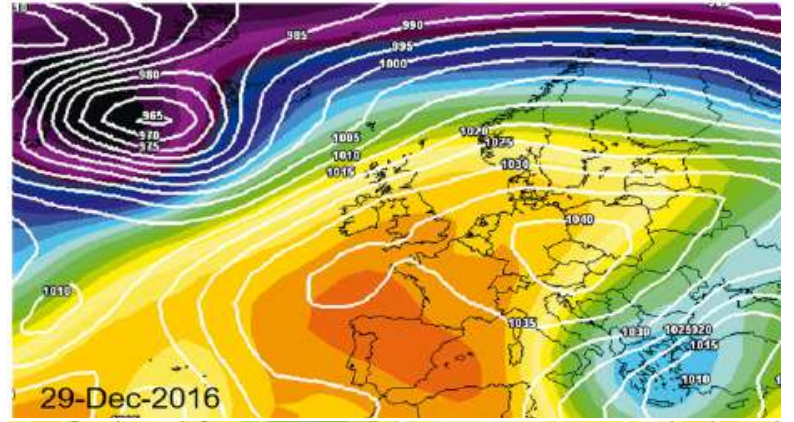

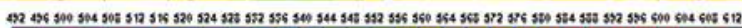

Fig. 1.- Altura geopotencial $(500 \mathrm{hPa})$ en dam geopotenciales (barra de colores) y presión al nivel del mar en hPa (isolíneas blancas) a las 12 UTC el 29 de diciembre de 2016 (Reanálisis NCEP). Obtenida de meteociel.fr

Durante el período navideño de 2016 (especialmente de 26 al 30 de diciembre), tuvo lugar un episodio de alta contaminación persistente en la ciudad de Madrid superándose, hasta en 86 ocasiones, valores promedios horarios de dióxido de nitrógeno de $200 \mu \mathrm{g} \cdot \mathrm{m}^{-3}$ en diferentes estaciones de calidad del aire de la ciudad (Borge et al., 2018). La superación de determinados umbrales en varias estaciones durante un número de veces implica la activación del protocolo del Ayuntamiento de Madrid, aprobado en 2016, mediante el cual se ponen en marcha mecanismos que sirven tanto para proteger la salud de los ciudadanos como para sensibilizar a la opinión pública. En función de los niveles alcanzados y la duración del episodio, se llevan a cabo medidas de restricción de tráfico en la ciudad y sus accesos para reducir estos niveles de contaminación y evitar que se alcance la situación de alerta. Durante el mencionado 
episodio de alta contaminación en Madrid, el día 29 de diciembre se llegó a activar el nivel 3 del protocolo (de un máximo de 4) en el que, entre otras medidas, se restringió por primera vez la circulación en el interior de la almendra central (área interior de la M30) del 50\% de la mayoría de los vehículos (control por matrícula par o impar). En este trabajo se analizan diferentes variables meteorológicas procedentes tanto de estaciones en superficie como de sondeos aerológicos del cercano aeropuerto Adolfo Suárez Madrid-Barajas, así como datos de calidad del aire (principalmente concentración de $\mathrm{NO}_{2}$ ) de la Red de Calidad del Aire del Ayuntamiento de Madrid. De acuerdo a los resultados obtenidos, la altura de la base de la inversión térmica obtenida a partir de los sondeos aerológicos a las 12 UTC, resultó ser un índice muy fiable de las condiciones meteorológicas desfavorables que llevan a poner en marcha el protocolo de actuaciones, ya que se encontró una alta correlación entre capas de mezcla muy someras a mediodía y picos muy altos de concentración de $\mathrm{NO}_{2}$ a lo largo de la tardenoche posterior.

Como resultado de este trabajo se propone que el análisis de la capa de mezcla prevista por los modelos de predicción se use como una herramienta útil para anticiparse a estos episodios de alta contaminación en las ciudades. El uso de los perfiles verticales del número de Richardson, que aporta información del nivel de turbulencia en la baja atmósfera, así como de la temperatura potencial, indicando la estabilidad de estratificación, permitiría además tener una buena caracterización de esta capa de mezcla. Valores de capa de mezcla en torno a $100 \mathrm{~m}$ al mediodía, más típicos en los meses del año con menor irradiación solar, deberían alertar sobre la posibilidad de sufrir eventos de contaminación atmosférica severos, y por lo tanto instar a tomar las medidas necesarias para una disminución de las emisiones. Éstas juegan un papel determinante en dichos episodios, por lo que son necesarias acciones realmente drásticas sobre ellas para que surtan efectos apreciables en la mejora de la calidad del aire.

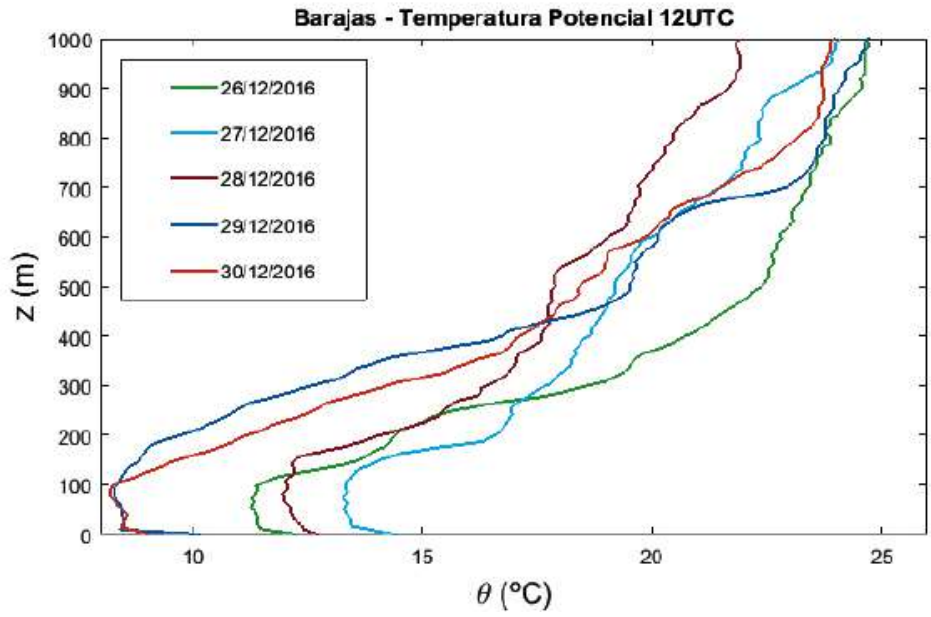

Fig. 2.- Perfiles verticales de temperatura potencial obtenidos de los sondeos de Madrid-Barajas a las $12 \mathrm{Z}$ del 26 al 30 de diciembre de 2016. Nótese cómo la capa de mezcla estos días ronda los $100 \mathrm{~m}$. Datos obtenidos de meteociel.fr 


\section{Referencias}

- Artıñano, B., Salvador, P., Alonso, D.G., Querol, X., Alastuey, A. 2003. Anthropogenic and natural influence on the PM10 and PM2.5 aerosol in Madrid (Spain). Analysis of high 519 concentration episodes. Environmental Pollution, 125, 453-465.

- Borge, R., Artíñano, B., Yagüe, C., Gomez-Moreno, F.J., Saiz-Lopez, A., Sastre, M., Narros, A., García-Nieto, D., Benavent, N., Maqueda, G., Barreiro, M., de Andrés, J.M., Cristóbal, A. 2018. Application of a short term air quality action plan in Madrid (Spain) under a high-pollution episode - Part I: Diagnostic and analysis from observations. Science of the Total Environment (In press). doi: 10.1016/j.scitotenv.2018.03.149

\section{Agradecimientos}

Este estudio ha sido financiado mediante el Proyecto TECNAIRE-CM (S2013/MAE2972) gracias a la Dirección General de Universidades e investigación de la Comunidad de Madrid. Asimismo, agradecemos al Ayuntamiento de Madrid y a AEMET la disponibilidad de sus datos. 